\title{
Biostimulation of Knee Cartilage Using Autologous Micro-Grafts: A Preliminary Study of the Rigenera Protocol in Osteochondral Lesions of the Knee
}

\author{
Agustín Dorta Fernández $^{1, ~ *}$, Ana Baroni Luengo ${ }^{2}$ \\ ${ }^{1}$ Hand Surgery and Microsurgery Unit, La Laguna, Spain \\ ${ }^{2}$ Department of Computer Science and Technology, International University of La Rioja, La Rioja, Spain \\ Email address: \\ dortafernandezagustin@gmail.com (A. D. Fernández), dranabaroni@gmail.com (A. B. Luengo) \\ ${ }^{*}$ Corresponding author
}

\section{To cite this article:}

Agustín Dorta Fernández, Ana Baroni Luengo. Biostimulation of Knee Cartilage Using Autologous Micro-Grafts: A Preliminary Study of the Rigenera Protocol in Osteochondral Lesions of the Knee. Rehabilitation Science. Vol. 3, No. 1, 2018, pp. 8-12.

doi: $10.11648 /$ j.rs.20180301.12

Received: September 26, 2017; Accepted: October 31, 2017; Published: January 23, 2018

\begin{abstract}
Osteochondral pathologies are associated with cartilage loss. Autologous micro-grafts, obtained from various tissues containing mesenchymal stem cells, has proven regenerative properties in various tissues. The study included patients with osteochondral pathology not responding to conventional therapies and considered suitable for treatment with autologous micro-graft. Fifteen days before the intervention, patients started treatment with chondroitin sulfate and supplementary vitamin $\mathrm{C}$ and zinc. Autologous micro-grafts were obtained from ear skin and cartilage, disaggregated using the Rigenera system, and injected into the knee under local anesthesia. After the intervention, patients underwent six-week rehabilitation program based on non-weight bearing exercises. Nine patients were enrolled into the study. The autologous graft transfer was successful in all patients, and no severe complications were recorded, including aesthetical defects in the donor site. Eight patients $(88.9 \%)$ experienced an improvement in all subdomains of the WOMAC scale. The median (IR) improvement in the WOMAC scale was $22.0(10.0$ - 40.0) points. The MRI examination revealed the absence of space-occupying lesions in the knee and the surrounding soft tissues in all patients. An edema reduction was observed in 8 of 9 patients (88.9\%); 3 patients $(33.3 \%)$ showed a thickening of the cartilage line. Autologous micro-graft transfer using the Rigenera system is safe and has promising results in the treatment of chondral injuries associated with pain and function limiting.
\end{abstract}

Keywords: Rigenera, Osteoartrhitis, Osteochondral Regions, Mesenchymal Stem Cells, Tissue Regeneration

\section{Introduction}

Osteochondral pathologies may result from trauma or sports injuries or be the consequence of disorders associated with cartilage degeneration, such as chondromalacia and osteoarthritis [1, 2]. Irrespective of the etiology of the osteochondral pathology, the loss of cartilage, evidenced in magnetic resonance images (MRI) as a thinning of the cartilage line, causes pain and physical disability [3-5]. In addition to surgery, the traditional management of degenerative cartilage diseases is based on physical therapy and pain relief. However, some patients do not respond well to pain relievers, and the regeneration of the damaged cartilage may last for a long time, thus compromising the complete functional recovery.

In the recent years, many efforts have been made in developing complementary strategies aimed at either regenerating the damaged cartilage or enhancing the natural regeneration process. In addition to the intra-articular use of hyaluronic acid, various approaches have been proposed to stimulate the production of new cartilage. Biostimulation techniques include physical approaches, such as the use of high-frequency biostimulation during surgery [6] or lowpower laser targeting the osteochondral defect [7,8], and biological approaches, such as autologous chondrocyte implantation [9] and the use of platelet-rich plasma to stimulate the production of new cartilage [10].

The finding of stem-like cell populations with multipotent features in various tissues has motivated the use of these cells 
for regenerative purposes $[11,12]$. However, the availability of ready-to-use methods for a safety and effective isolation of mesenchymal stem cells during surgery has been a bottleneck for the clinical use of multipotent cells. The Rigenera protocol is a novel strategy for tissue mechanical disaggregation which allows obtaining autologous micrografts enriched with mesenchymal stem cells during surgery $[13,14]$. Compared to enzymatic techniques, mechanical disaggregation has claimed to be faster and safer. To date, the Rigenera protocol has shown clinical efficacy in the management of complex wounds $[15,16]$ and the regeneration of the dental pulp in periodontal surgeries (Periodontal tissue generation) [17]. However, the identification of stem-like cells in many other tissues suggests additional uses of autologous micro-graft preparation and transfer [12]. In this preliminary study, the clinical and functional outcomes of cartilage autologous micro-grafting (obtained with the Rigenera protocol) in patients with knee osteoarticular manifestation of cartilage injury or degeneration is described.

\section{Materials and Methods}

\subsection{Study Subjects}

In this prospective study, patients with mechanical and/or degenerative osteochondral pathology who did not respond to conventional therapies and were considered suitable for being treated with the autologous micro-grafts in an experimental setting were included. Patients were recruited and treated by the same surgeon between January 2016 and April 2016.

In addition to the complete blood count and the erythrocyte sedimentation rate, all patients were screened for immune system diseases and metabolic abnormalities, including vitamin D3 and B12, triglycerides, cholesterol, glycated hemoglobin, and thyroid hormone levels. Testosterone levels were also assessed in male subjects. Patients with immune or inflammatory diseases such as familial osteoarthritis, history of rheumatic inflammatory diseases, and those with a positive antinuclear antibody test, the presence of rheumatoid factor, and anti-streptolysin $\mathrm{O}$ greater than 150 were excluded. Patients with morbid obesity, diabetes mellitus, and other metabolic diseases - including those treated with statins and/or hypolipidemic agents - were also excluded. All patients signed the informed consent to undergo the experimental procedure.

\subsection{Autologous Micro-Grafts Transfer}

15 days before the intervention, patients started treatment with chondroitin sulfate and supplementary vitamin $\mathrm{C}$ and zinc. Patients with low levels of vitamin D3 and $\mathrm{B}_{12}$ were also supplemented with these vitamins. Non-steroidal antiinflammatory drugs were not allowed during the 15 days preceding the intervention. Six hours before the intervention, patients started treatment with azithromycin $500 \mathrm{mg}$ (maintained during the two days following the intervention) and fasted until intervention.

The cartilage graft was obtained from the auricle cartilage of the ipsilateral ear to the target knee using $\times 3.5$ magnification Zeiss lens in sterile file. To expose the auricle cartilage, an eye-shaped skin graft of 2 x $1.2 \mathrm{~cm}$ (approximately $120 \mathrm{~mm}^{2}$ ) was removed. The cartilage graft was excised with a scalpel (No. 11) and microsurgery scissors. The perichondrial tissue surrounding the cartilage gap was scratched and added to the sample. The wound was rinsed with a $1: 1$ solution of $3 \%$ hydrogen peroxide and saline and sutured with 6/0 subcutaneous Vicryl and 2-to-3 transverse stitches.

Approximately $0.1 \mathrm{~cm}^{3}$ of the dermis and subcutaneous tissue were detached from the skin graft and mixed with 0.25 -to- $0.3 \mathrm{~cm}^{3}$ of auricle cartilage and perichondrial tissue. The sample was mechanically disaggregated using the Rigenera protocol according to the manufacturer's specifications during two cycles of 2 minutes, obtaining a cellular graft of $4 \mathrm{~cm}^{3}$. The final number of cells obtained was not determined. However, according to previous experiences with the Rigenera system, the nasal cartilage yielded $9088.30 \pm 788.86$ chondrocytes per $\mathrm{mL}$ of digested tissue [22]; hence, the sample was expected to yield 2-to$3 \times 10^{3}$ chondral cells. Likewise, the phenotype of dermal cells obtained using the Rigenera system was previously analyzed by Svolacchia et al., who found the following percentages of mesenchymal stem cells markers: $82 \%$ of CD34, $82 \%$ of CD90 (Thy-1), and 53\% of CD105 [23]. The authors reported a yield of $80 \times 103$ cells $-70-80 \%$ of them mesenchymal - with $92 \%$ of viability from $2 \mathrm{~mm} 3$ of dermal tissue. Considering that dermal sample was approximately $120 \mathrm{~mm} 3$ (120-mm2 surface and 1-mm thickness), to obtain nearly $4.8 \times 105$ cells was expected.

Disaggregated cells were finally injected into the knee by using a patellar injection under local anesthesia. Passive knee movement was applied to evenly distribute the sample inside the knee.

\subsection{Rehabilitation Protocol and Assessments}

Full weight-bearing without any orthosis was allowed six weeks after intervention. During this period, patients were advised to perform non-weight bearing exercises involving the hamstring and quadriceps, as well as stationary cycling without resistance. Upon healing of the ear wound, patients were allowed to swim. Normal gait was achieved through progressive weight bearing for six additional weeks. Patients were not allowed to take non-steroidal anti-inflammatory drugs during the entire follow-up.

The progress of osteochondral injury was assessed by MRI tests, which were performed before and twelve weeks after the intervention. Functional and pain assessments were performed using the WOMAC scale (Spanish version) [24, 25]. Data is presented as raw and as the median and interquartile range (IR, corresponding to percentiles 25 and $75)$ or the mean and standard deviation (SD). 


\section{Results}

\subsection{Patients and Procedure}

The autologous graft transfer was performed in nine patients meeting all the selection criteria. The mean (SD) age was 51.7 (6.2), 56\% were male, and none of them were active smokers. In all cases, the procedure was successful, and none of them experienced infections or severe complications after the procedure. Two patients experienced joint effusion, manifested at a few days after the procedure. No aesthetical defects were observed at the donor site in any patient and none of them complained about the result on the donor site. One patient with a history of blood dyscrasias experience retroauricular bruising which healed without other interventions than vitamin $\mathrm{K}$ supplementation and any aesthetical defect.

\subsection{Symptoms and Functional Outcome}

Except one patient who experienced edema associated with cell infiltration, all patients referred a remarkable pain decrease during the few days following the procedure. At the end of the follow-up all patients all patients returned to their activities performed before the worsening of the cartilage injuries. Table 1 shows the details of the functional and pain assessment at baseline and the end of the follow-up. Eight patients $(88.9 \%)$ experienced an improvement in all subdomains of the WOMAC scale. Median (IR) total WOMAC score in the study population was 39.0 (19.5 59.0) before intervention and $9(3.0-23.5)$, a median improvement of $22.0(10.0-40.0)$ points. Patient 3 improved her stiffness and functional impairment but worsened pain associated with her knee condition. Overall, the median (IR) pain, stiffness, and function improvement were 5.0 (3.3 9.0) on a 20-point scale, $3.0(1.3-3.8)$ on an 8-point scale, and $19.5(9.3-30.0)$ on a 68 -point scale, respectively.

The MRI examination revealed the absence of spaceoccupying lesions in the knee and the surrounding soft tissues in all patients. An edema reduction was observed in 8 of 9 patients $(88.9 \%)$ (Figure 1A). The woman in case 9 displayed more edema in the post-intervention than in the pre-intervention MRI. Chondromalacia showed a partial regression in 5 of 7 cases $(71.4 \%)$ in which the progress of the chondromalatic signs was reported. Three patients $(33.3 \%)$ showed a thickening of the cartilage line (Figure
1B). Of 8 patients with visible cartilage tear at baseline, 4 maintained the injury, 1 showed a remarkable improvement, and 2 showed no signs of the injury at the post-intervention MRI. Three patients showed a Baker's cyst at baseline: one remained unchanged, one reduced the cyst size, and one had no visible cysts at post-intervention MRI. The patient who experienced a worsening in knee stiffness had a cartilage injury before the procedure which was maintained after the procedure.

Figure captions
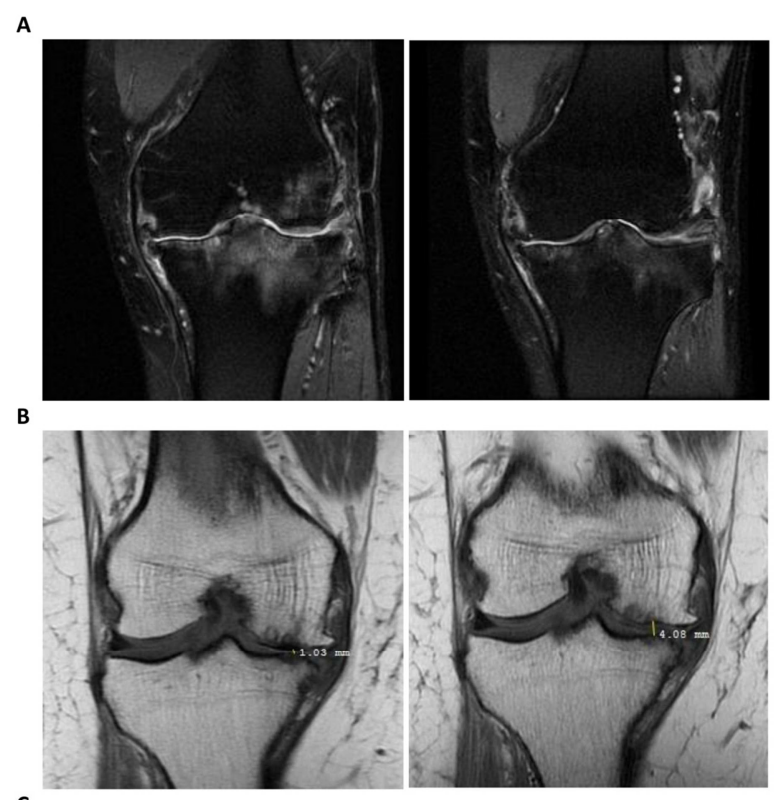

C

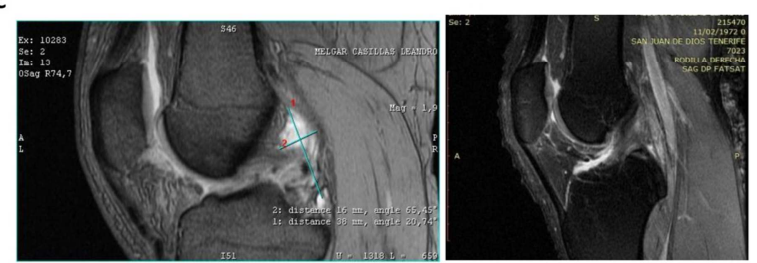

Figure 1. Highlights of MRI findings before and after procedure. A: case 4 at presentation (left) and sixteen weeks after (right); the images show an edema reduction and an increase in the definition of the cartilage line. B: case 7 at presentation (left) and twelve weeks after the intervention (right); the cartilage thickness experienced an increase of $2.78 \mathrm{~cm}$. C: case 1 at presentation (left) and 17 weeks after; the Baker's cyst of $36 \mathrm{~mm}$ length $\times 16$ $\mathrm{mm}$ wide identified at presentation showed a complete remission 17 weeks after the procedure.

Table 1. Patient characteristics and results of the WOMAC scale.

\begin{tabular}{|c|c|c|c|c|}
\hline \multirow[b]{2}{*}{ Patient no. } & \multicolumn{4}{|c|}{ WOMAC scores* pain | stiffness | function limitation } \\
\hline & Sex & Age (years) & Before the intervention & After the intervention \\
\hline 1 & male & 44 & $4|1| 8$ & $0|0| 0$ \\
\hline 2 & male & 44 & $2|3| 4$ & $0|0| 2$ \\
\hline 3 & female & 54 & $3|5| 19$ & $7|2| 15$ \\
\hline 4 & male & 63 & $11|5| 45$ & $6|2| 18$ \\
\hline 5 & male & 50 & $7|2| 30$ & $4|2| 17$ \\
\hline 6 & male & 48 & $12|6| 29$ & $3|2| 4$ \\
\hline 8 & female & 56 & $12|5| 40$ & $3|3| 9$ \\
\hline 9 & female & 50 & \begin{tabular}{l|l|l}
6 & 3 & 17 \\
\end{tabular} & \begin{tabular}{l|l|l}
1 & 0 & 3 \\
\end{tabular} \\
\hline
\end{tabular}

*Score ranges for the assessment of pain, stiffness, and functional limitation are $0-20,0-8$, and 0-68, respectively. 


\section{Discussion}

We present here in 9 cases of patients with cartilage injuries who experienced an improvement in pain and function - some of them with changes visible at MRI examination - after intra-articular injection of an autologous micro-graft obtained with the Rigenera protocol.

Chondral injuries in the knee are a challenging scenario for surgical reconstruction. Despite the good results observed with prosthetic resurfacing, there is increasing interest in developing safety and effective procedures for biologic resurfacing and joint preservation [18]. The rationale behind the Rigenera protocol lies in the multipotent properties of the mesenchymal stem cells which may promote cell differentiation and subsequent new cartilage formation $[13,14]$. In our experience, the use of autologous grafts enriched in mesenchymal cells resulted in healing or improvement of cartilage defects visible at MRI examination. After graft transfer intervention, all patients except one showed an edema reduction. However, considering the function and pain improvement in this patient, the post-intervention edema was attributed to the normal healing process. Most patients had a visible cartilage tear or decrease in the cartilage line at baseline. The technical limitations of the MRI assessment did not allow for a quantification of interesting parameters - such as the cartilage thickness or the size of cartilage defects - in all patients. Despite this limitation, three patients showed a remarkable increase in cartilage thickness (for 1.03 to 4.08 $\mathrm{cm}$ in the best case observed) at the examinations before and after the intervention. It is noteworthy that one patient had a 36-cm length Baker's cyst at baseline examination, which was not visible at MRI examination after the intervention, indicating a significant reduction of the inflammation.

Regardless of the MRI findings, all patients except one showed an improvement in pain, stiffness, and function, and returned to their regular physical activity twelve weeks after the intervention. In some cases, the cartilage defect had progressed to a severe function limitation associated with intense pain which prevented patients from performing their activities of daily living, including work and normal gating. Pain and function were assessed by means of the Spanish version of the WOMAC scale, which has been extensively used in research and in routine clinical practice for assessing the severity of knee injuries [19]. The results of the WOMAC assessment in studies investigating the efficacy of other biostimulation techniques are heterogeneous, with studies reporting significant improvement and other reporting a slight worsening at 2 or 3 months [10, 20]. Also, the different study designs and etiologies hamper reliable comparisons between studies. However, without losing sight of these limitations, in the study patients showed a greater trend towards functional and pain improvement than those treated with platelet-rich plasma preparations and assessed with the same scale. Furthermore, although the number of included patients does not allow for a correlation analysis, pain improvement concurred with an improvement in function and stiffness, and the WOMAC scores were overall consistent with the MRI findings, as expected, considering the correlation between pain and cartilage defects visible at MRI examination [21].

The strict selection criteria limited the number of patients included in the study. However, as this was an exploratory test of safety and effectiveness of the Rigenera protocol in the management of knee cartilage conditions, we considered the exclusion of immune and inflammatory diseases an important condition to achieve the best performance of the transferred cells and to assess safety independently. Another important limitation was the heterogeneity of the MRI information, which precluded a quantitative analysis of the MRI findings. However, MRI assessments provided valuable input on the physiological effects of cartilage biostimulation.

In summary, this study shows that the transfer of autologous micro-grafts using the Rigenera protocol is safe and has promising results in the treatment of cartilage injuries associated with pain and functional limiting. These results suggest the suitability of further prospective, controlled studies with larger cohorts to confirm the therapeutic properties of this intervention.

\section{References}

[1] Chan VO, Moran DE, Mwangi I, Eustace SJ. Prevalence and clinical significance of chondromalacia isolated to the anterior margin of the lateral femoral condyle as a component of patellofemoral disease: Observations at MR imaging. Skeletal Radiol. 2013 Aug 21; 42(8):1127-33.

[2] Kao YJ, Ho J, Allen CR. Evaluation and management of osteochondral lesions of the knee. Phys Sportsmed. 2011 Nov $13 ; 39(4): 60-9$.

[3] Wang J, Han W, Wang X, Pan F, Liu Z, Halliday A, et al. Mass effect and signal intensity alteration in the suprapatellar fat pad: Associations with knee symptoms and structure. Osteoarthr Cartil. 2014 Oct; 22(10):1619-26.

[4] Lankhorst NE, Damen J, Oei EH, Verhaar JAN, BiermaZeinstra SMA, van Middelkoop M. Incidence, prevalence, natural course and prognosis of patellofemoral osteoarthritis; data of cohort hip and cohort knee study. Osteoarthr Cartil. 2015 Dec; 23(2011):A42-3.

[5] Anandacoomarasamy A, Smith G, Leibman S, Caterson I, Giuffre B, Fransen M, et al. Cartilage defects are associated with physical disability in obese adults. Rheumatology (Oxford). 2009 Oct; 48(10):1290-3.

[6] Monllau JC, Leal J, Voss C, Pelfort X, Tey M, Pavlovich RI. Good Outcome After Meniscal Repair Using an All-Inside Suturing System in Combination with High-Frequency Biostimulation. Lindeque BGP, editor. Orthopedics. 2010 Jun 1; 33(6):407-12.

[7] Torricelli P, Giavaresi G, Fini M, Guzzardella GA, Morrone $\mathrm{G}$, Carpi A, et al. Laser biostimulation of cartilage: In vitro evaluation. Biomed Pharmacother. 2001 Mar; 55(2):117-20. 
[8] Morrone G, Guzzardella GA, Torricelli P, Rocca M, Tigani D, Brodano GB, et al. Osteochondral lesion repair of the knee in the rabbit after low-power diode Ga-Al-As laser biostimulation: an experimental study. Artif Cells Blood Substit Immobil Biotechnol. 2000 Jul; 28(4):321-36.

[9] Macmull S, Jaiswal PK, Bentley G, Skinner JA, Carrington RWJ, Briggs TWR. The role of autologous chondrocyte implantation in the treatment of symptomatic chondromalacia patellae. Int Orthop. 2012 Jul; 36(7):1371-7.

[10] Marmotti A, Rossi R, Castoldi F, Roveda E, Michielon G, Peretti GM. PRP and articular cartilage: a clinical update. Biomed Res Int. 2015; 2015:542502.

[11] Goodell BMA, Brose K, Paradis G, Conner AS, Mulligan RC. Isolation and Functional Properties of Murine Hematopoietic Stem Cells that are Replicating In Vivo By Margaret A. Goodell, Katja Brose, Glenn Paradis,* A. Stewart Conner,* and Richard C. Mulligan. 1996; 183(April).

[12] De Francesco F, Romano M, Zarantonello L, Ruffolo C, Neri $\mathrm{D}$, Bassi $\mathrm{N}$, et al. The role of adipose stem cells in inflammatory bowel disease: From biology to novel therapeutic strategies. Cancer Biol Ther. 2016 Sep 14; 17(9):889-98.

[13] Trovato L, Monti M, del Fante C, Cervio M, Lampinen M, Ambrosio L, et al. A New Medical Device Rigeneracons Allows to Obtain Viable Micro-Grafts From Mechanical Disaggregation of Human Tissues. J Cell Physiol. 2015 Oct; 230(10):2299-303.

[14] Purpura V, Bondioli E, Graziano A, Trovato L, Melandri D, Ghetti $M$, et al. Tissue Characterization after a New Disaggregation Method for Skin Micro-Grafts Generation. J Vis Exp. 2016 Mar 4;(109):e53579.

[15] Giaccone M, Brunetti M, Camandona M, Trovato L, Graziano A. A new medical device, based on Rigenera protocol, in the management of complex wounds. J Stem Cells Res. Rev Rep. 2014; 1(3):3.

[16] Marcarelli M, Trovato L, Novarese E, Riccio M, Graziano A. Rigenera protocol in the treatment of surgical wound dehiscence. International Wound Journal. 2016 Apr; 277-81.
[17] Graziano A, Carinci F, Scolaro S, D'Aquino R. Periodontal tissue generation using autologous dental ligament micrografts: case report with 6 months follow-up. Ann Oral Maxillofac Surg. 2013; 1(2):20.

[18] Capeci CM, Turchiano M, Strauss EJ, Youm T. Osteochondral Allografts. Applications in Treating Articular Cartilage Defects in the Knee. Bullettin th Hosp Jt Deseases. 2013; 71(1):60-70.

[19] Bellamy N, Buchanan WW, Goldsmith CH, Campbell J, Stitt LW. Validation study of WOMAC: a health status instrument for measuring clinically important patient relevant outcomes to antirheumatic drug therapy in patients with osteoarthritis of the hip or knee. J Rheumatol. 1988; 15(12):1833-40.

[20] Metcalf KB, Mandelbaum BR, McIlwraith CW. Application of Platelet-Rich Plasma to Disorders of the Knee Joint. Cartilage. 2013 Oct; 4(4):295-312.

[21] Baum T, Joseph GB, Arulanandan A, Nardo L, Virayavanich W, Carballido-Gamio J, et al. Association of magnetic resonance imaging-based knee cartilage t 2 measurements and focal knee lesions with knee pain: Data from the osteoarthritis initiative. Arthritis Care Res. 2012 Feb; 64(2):248-55.

[22] Gentile P, Scioli MG, Bielli A, Cervelli V. A combined use of Chondrocytes Micro Grafts (CMG) Mixed with Platelet Rich Plasma (PRP) in Patients Affected by Pinch Nose Deformity. J Regen Med. 2016; 2016.

[23] Svolacchia F, De Francesco F, Trovato L, Graziano A, Ferraro GA. An innovative regenerative treatment of scars with dermal micrografts. J Cosmet Dermatol. 2016.

[24] Batlle-Gualda E, Esteve-Vives J, Piera M, Hargreaves R, Cutts J. Adaptación transcultural del cuestionario WOMAC específico para artrosis de rodilla y cadera. Rev Española Traumatol. 1999; 26:38-45.

[25] Escobar A, Quintana JM, Bilbao A, Azkárate J, Güenaga JI. Validation of the Spanish Version of the WOMAC Questionnaire for Patients with Hip or Knee Osteoarthritis. Clin Rheumatol. 2002 Nov 1; 21(6):466-71. 